\title{
Jovian electrons and magnetic traps with inner acceleration regions
}

\author{
K. Kecskeméty ${ }^{1}$ \\ Wigner Research Centre for Physics, Budapest, Hungary \\ E-mail: kecskemety.karoly@wigner.mta.hu
}

\section{E. I. Daibog, L.L. Lazutin, Yu.I. Logachev}

Lomonosov Moscow State University, D.V. Skobeltsyn Nuclear Physics Research Institute, Moscow, Russia

\section{J. Kóta}

Lunar and Planetary Laboratory, University of Arizona, Tucson, USA

Simultaneously with 27-day variations of MeV Jovian electrons, observed during the deep solar minimum in 2007-2008 in 14 consequent solar rotations, also short duration (2-3 days) enhancements of the fluxes of 0.1-1 MeV electrons and protons were registered. These enhancements took place during each solar rotation simultaneously at SOHO (observed by COSTEP/EPHIN and LION) and ACE (EPAM) and appeared earlier and later at STEREO B and STEREO A (IMPACT/SEPT), respectively, usually before the flux of high-energy Jovian electrons started to rise, so the lower energy peaks did not coincide with those maxima. The Sun was extremely quiet throughout the whole period considered (no $10.7 \mathrm{~cm}$ radio or soft X-ray emission) and could not be the origin of these low energy particles. We consider the hypothesis that a magnetic trap with trapped Jovian electrons, corotating with the Sun, had some specific regions «ridges» - with enhanced level of turbulence inside capable of accelerating electrons and protons up to 1 $\mathrm{MeV}$. The formation of such «a ridge» is natural at the front part of the trap; differently located accelerating regions may appear due to the penetration of new field lines into the trap. These ridges appear to be rather stable, much of them are observed to survive a few solar rotations, disappear and appear again, initiating series of short-living enhancements of low energy electrons and protons, separated by 27-day intervals. According to this hypothesis, the electrons registered during the 2007-2008 solar activity minimum have two components: (a) periodic 27-day gradual Jovian-originated variations; (b) quasi-periodic short (2-3 days) increases of low-energy electrons and protons, accelerated directly inside the magnetic trap. Numerical simulations to model the propagation of energetic protons and electrons in the presence of such magnetic configuration are presented.

The 34th International Cosmic Ray Conference

30 July- 6 August, 2015

The Hague, The Netherlands

\footnotetext{
${ }^{1}$ Speaker

(c) Copyright owned by the author(s) under the terms of the Creative Commons Attribution-NonCommercial-ShareAlike Licence.
} 


\section{Introduction}

Regular variations of fluxes of different energy charged particles with approximately 27day periodicity may originate from different sources associated with the Sun. These are corotating particles of solar origin directly, or those arising from closed structures of the interplanetary magnetic field filled with particles of non-solar origin, e.g. those emitted by the magnetosphere of Jupiter [1-3], or accelerated just inside these structures. Jovian electrons represent a perfect tool to investigate the structure of inner heliosphere. They propagate from Jupiter to the Earth along interplanetary magnetic field lines and can be trapped in closed magnetic structures,

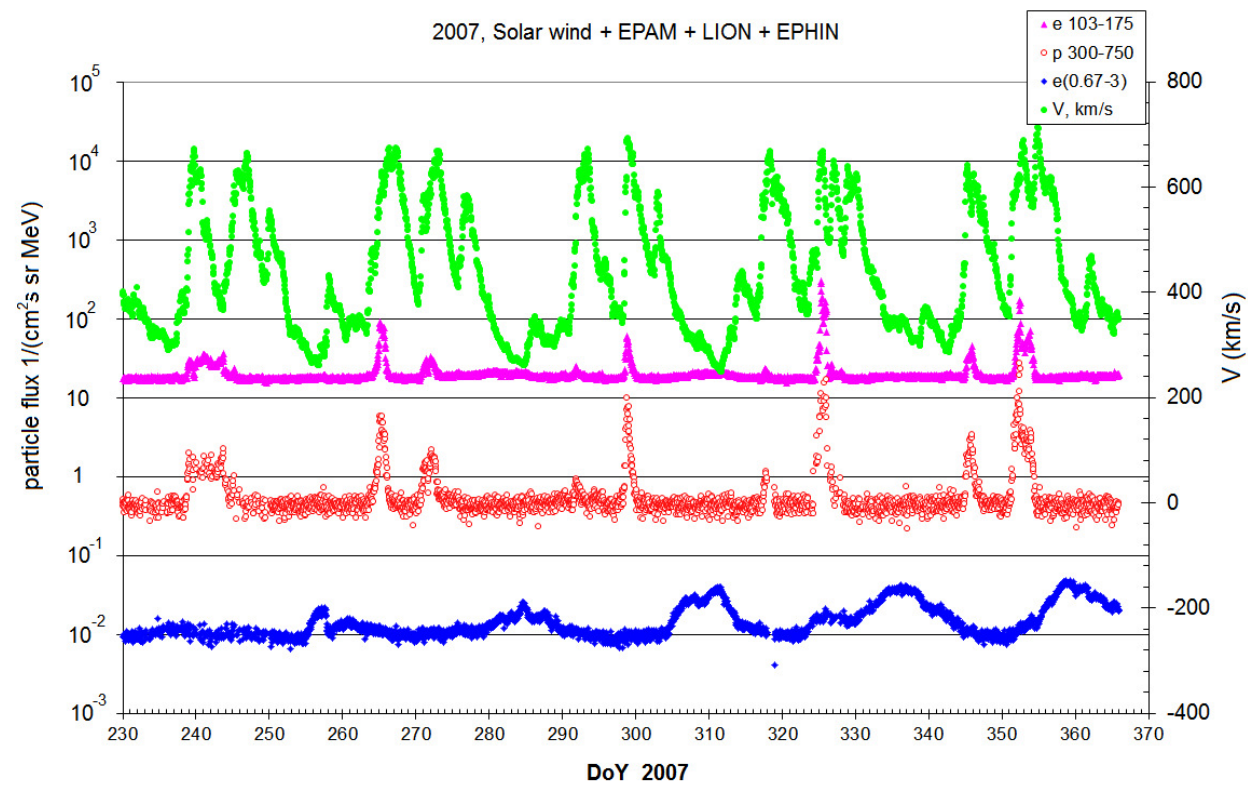

Figure 1. Fluxes of energetic 0.7-3 MeV electrons (SOHO/EPHIN) and solar wind speed at $1 \mathrm{AU}$ (OMNIWeb) together with low energy electron (ACE) and protons (SOHO) fluxes during the solar activity minimum of 2007-2008.

co-rotating with the Sun. In both cases judging from the flow dynamics it is possible to infer the structure of the magnetic field in the interplanetary space. In this paper, based on energetic electron and ion observations, we study the properties of the magnetic trap connecting Jupiter with the immediate neighborhood of the Sun.

Figure 1 demonstrates the existence of repeating structures in the solar wind. A nearly perfect similarity of these structures was observed during the first six solar rotations. At the end of this period the structure slightly changed, but the trap was not destroyed, which is confirmed by the existence of repeating nearly 27-day variations of $\mathrm{MeV}$ electrons at the lower panel. Long living solar wind streams of different speeds in the interplanetary space, carrying solar magnetic field, create closed structures of different volumes - CIRs and other inhomogeneities of magnetic field. These structures may trap particles, brought there in any way. In some cases such magnetic traps co-rotate with the sun and exist as long as the stationarity of the solar wind, which created this magnetic field configuration, is maintained (see Figure 2). 


\section{Magnetic traps}

Figure 2a shows the schematic view of a trap, extending up to 2-3 AU from the Sun. Traps of such a size usually do not contain charged particles and no flux increases are observed at the Earth. In this case magnetic traps were created by field lines associated with long-living equatorial coronal holes, whose configuration varies slightly from one to another solar rotation depending on the differential rotation of convection zone of the sun (and depends on the differential rotation of convection zone of the sun). Figure $2 b$ shows a trap extending beyond the orbit of Jupiter. Such long living trap, passing by Jupiter, may be filled with energetic electrons, accelerated in the Jovian magnetosphere, co-rotating with the Sun and would carry trapped electrons everywhere between Sun and Jupiter including the vicinity of the Earth. Such a trap must satisfy a few essential conditions. These are large extent and long-term stability. Beyond the Jupiter orbit it must have a magnetic mirror to prevent free escape of electrons. The interior of the trap is expected to have enhanced magnetic field.
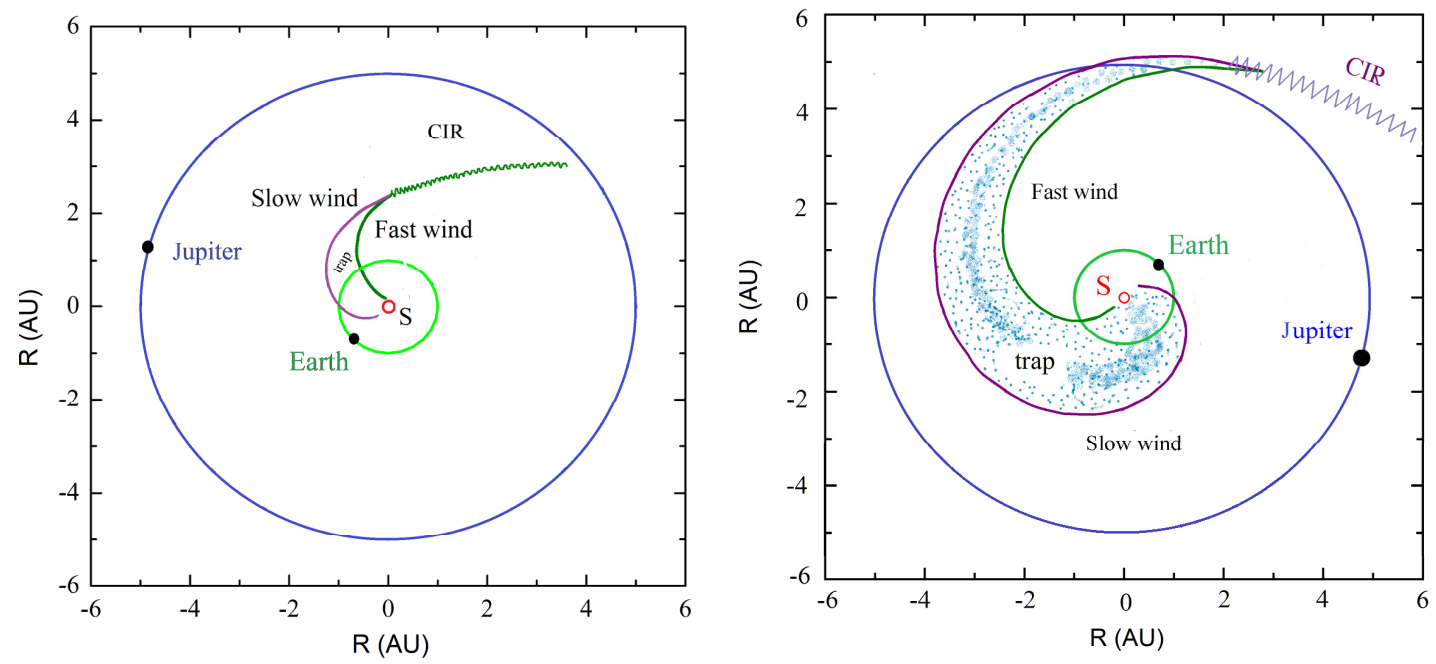

Figure 2. Schematic view of a magnetic trap, filled with electrons under every passage by Jupiter. Left panel: a relatively small sized empty trap, right panel: a well-developed trap already has passed by Jupiter and is filled with energetic electrons.

During the exceptionally deep minimum of solar activity between 2007 and 2009 the Sun was characterized by extremely quiet space weather, the structure of the solar wind was very stable, allowing the formation of long-living large magnetic traps (Figure 2b) filled with Jovian electrons, as registered at SOHO (EPHIN). The registration of Jovian electrons is possible by instruments with low background under the absence of disturbances at the Sun and in the interplanetary space.

\section{Conditions and lifetime of traps}

Closed structures of magnetic field, forming charged particle traps, appear practically incessantly in the inner heliosphere. The magnetic field is stronger near the Sun and represents a mirroring element for charged particles. At the opposite, far side of the trap the mirror is formed as a result of interaction between fast and slow solar wind streams. Variations of solar wind 
eventually destroy these traps, but new ones are forming and so on. The long time existence of stationary structures of solar wind promotes the formation of long-living traps filled with charged particles. The longest lifetime of magnetic trap was observed during the solar minimum of 2007-2008 and extended to 14 successive solar rotations.

In the solar equatorial plane the shape of the trap is defined by magnetic field lines Archimedean spirals; its latitudinal size extends probably up to 30-40 degrees, where there is no appreciable change of the solar wind speed according to Ulysses data. The time profiles of Jovian electrons in each of the 14 solar rotations of 2007-08 reached local maxima closer to the leading front of the trap and gradually decreased towards its rear edge. This shape also indicates that the lifetime of particles in the trap can exceed one solar rotation. The long lifetime may occur also as a result of acceleration of particles by turbulent magnetic field inside the trap,

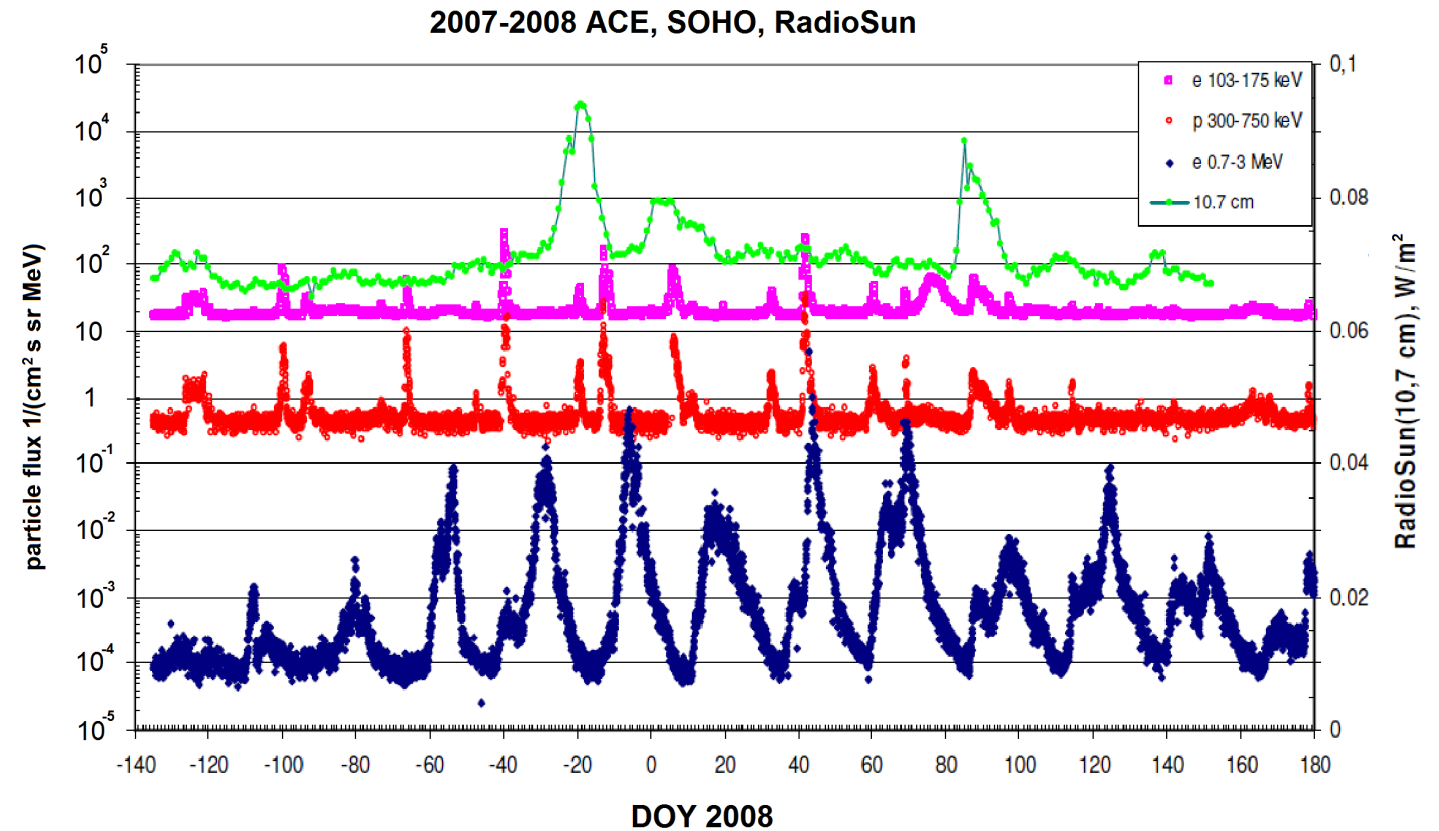

Figure 3. Jovian electrons and accompanying bursts of low-energy electrons and protons of non- solar origin, which follow from the absence of radio bursts during most part of this time interval.

which is natural under interaction of different speed solar wind streams. Favorable conditions for registration Jovian electrons existed also during other quite solar activity periods and they were registered during practically all activity minima by Pioneer 10, 11, IMP-8 and other spacecraft. One of such periods, in 1995-96, according to simultaneous IMP-8 (CPME) and SOHO (LION) data is presented in Figure 5.

Figure 3 shows bursts of electrons and protons, associated neither with Jovian electrons nor with solar activity in 2007-2008. As the figure demonstrates, significant fluxes of electrons (45-300 keV, ACE/EPAM) and protons (300-750 keV, SOHO/LION) were registered during each solar rotation under every passage of the trap by the s/c. The flux increases were short, lasting for about only 2-3 days, and started before the beginning of the increase of Jovian electrons. These low energy electrons and protons were not flare originated, the Sun was extremely quiet throughout the whole period considered (neither $10.7 \mathrm{~cm}$ radio, nor soft X-ray 
disturbed emission, was observed, see Figs.3 and 4) and could not be the origin of these lowenergy particles. Their profiles were not typical to SEP events (impulse-formed). In addition, the independent regular appearance of accelerated low-energy electrons and protons simultaneously with energetic Jovian electrons is very unlikely. The non- solar origin of low energy particles and their connection to the structures, co- rotating with the sun is confirmed be the STEREO-A and B (see Figure 4). The bursts were observed in order, corresponding to the location of s/c: STEREO-B (SEPT- e 105-125 keV), ACE \{( EPAM - e 103-175 keV), and SOHO (LION - p $300-750 \mathrm{keV}$ ) and STEREO-A (SEPT- e 105-125 keV).

\section{ACE/EPAM, SOHO/COSTEP, STEREO A+B/SEPT}

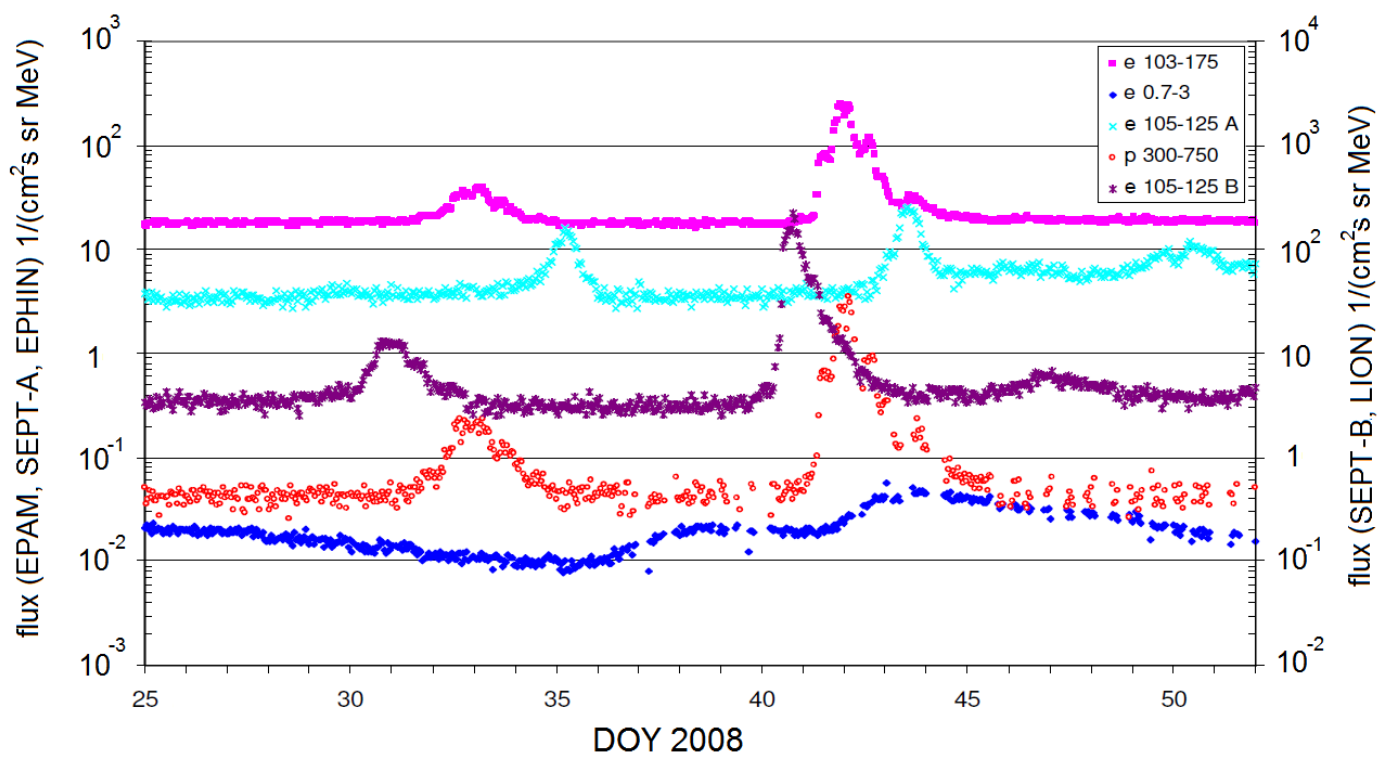

Figure 4. Confirmation of spatial nature of electron and proton bursts. Particle fluxes measured during one solar rotation between January 25 and February 21, 2008 with a marked shift of observed e and $\mathrm{p}$ bursts at different spacecraft under very quiet sun.

It is most plausible to regard these flux increases as linked to the magnetic trap with highly compressed magnetic field. We interpret these observations as the existence of compressed turbulized ridges of magnetic field at the leading front of the trap, giving rise to constant acceleration of electrons and protons up to $<1 \mathrm{MeV}$. They indicate the complexity of the trap structure with ridges inside the trap. Near the center of the trap, corresponding to the maximum flux of Jovian electrons, practically no such ridges were observed.

Figure 5 presents fluxes of Jovian electrons and $0.3-0.75 \mathrm{MeV}$ protons from $\mathrm{SOHO}$ along with the GOES soft X-ray flux during the 1995-1996 solar activity minimum. This picture is similar to the 2007-2008 period though not at such quite sun - soft X-ray bursts were observed during every solar rotation, but fluxes of Jovian electrons are not associated with weak active processes at the Sun initiating only bursts of soft X-rays. 


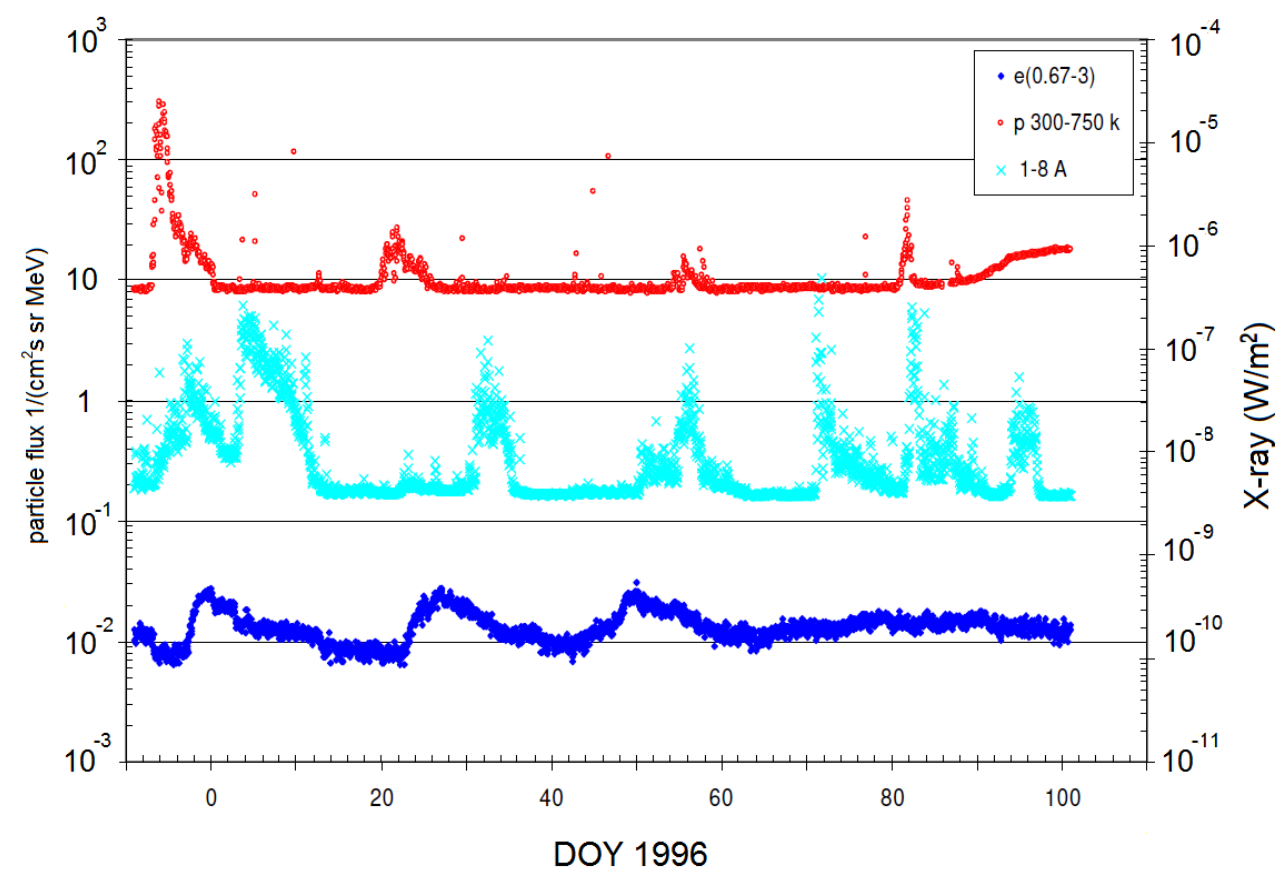

Figure 5. Solar activity minimum of 1996. Jovian electrons (IMP-8, CPME), low energy protons (SOHO, LION) and soft X-rays (OMNIWeb). Neither electrons nor protons are connected with active processes at the Sun. Proton bursts do not coincide with maxima of J- electron fluxes, suggesting different origin of these particles.

\section{Simulation}

Corotating interaction regions (CIRs) are formed by the interaction between high and slow solar wind plasma due to the tilted solar magnetic dipole and solar rotation. 27-day waves in galactic cosmic ray (GCR) flux is interpreted as a result of quasi-periodic variations in the solar wind speed and IMF. CIRs are known to accelerate energetic particles at the pair of Forward/Reverse shocks or at regions where the plasma is being compressed but the shocks has not been formed yet [4]. The numerical model presented here is similar to that used in [5,6], where Jupiter is a moving source in the co-rotating frame and perfect co-rotation is assumed. The values of solar wind speed and magnetic field are specified near the Sun (the solar wind had two high-speed streams about 8 days apart, roughly reproducing the 1 AU speed profiles in Fig. 1 but not fitted to match observations precisely). The Parker equation was solved in the corotating frame, and it produced a combined effect of the CIR and magnetic connection. The solar wind profile was not fitted to reproduce precise observations.

Figures $6 \mathrm{a}$ and $\mathrm{b}$ display the solar wind density and Jovian electron flux; Figure 7 shows the resulting Jovian electron fluxes as a function of time. This can be understood as during preferable phase of the CIR with respect to Jupiter, the stronger magnetic field behind the planet prevents the electrons from escaping the region. [7] had a similar observation for galactic cosmic rays, i.e. that high GCR fluxes appear during high plasma density - and B, fully correlating with it. 

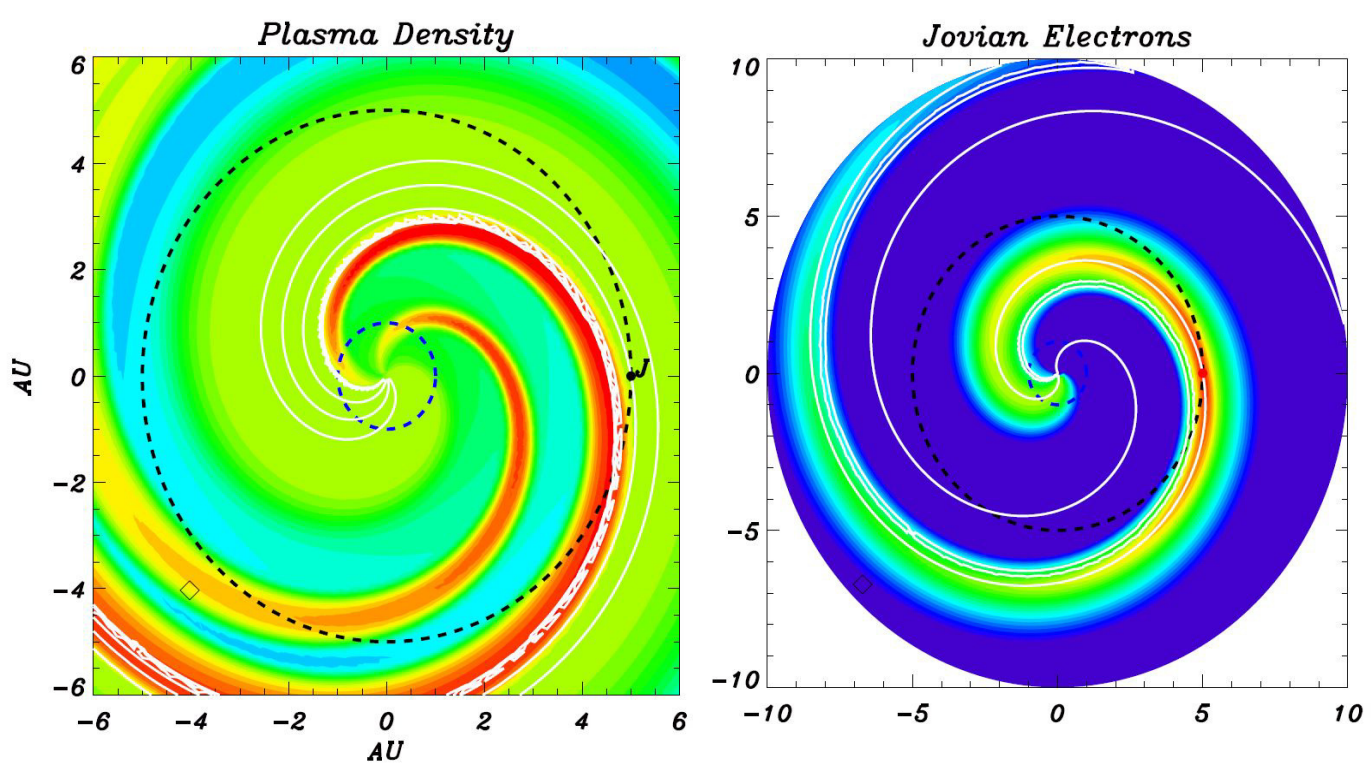

Figure 6. Contour plots of solar wind density (left) and Jovian electron flux of the CIR model with the orbits of Earth and Jupiter. Thin lines indicate spiral magnetic field lines.

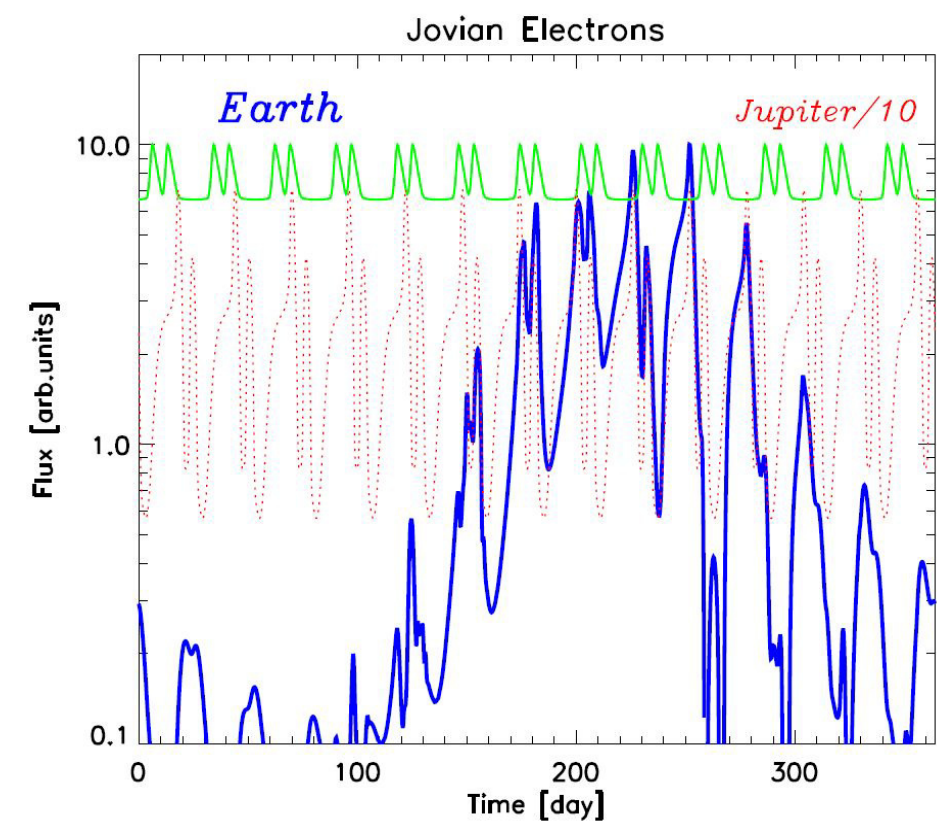

Figure 7. Time evolution of simulated plasma speed at Earth (green) together with Jovian electron flux at Earth (solid) and near Jupiter (dotted).

\section{Summary}

Short (2-3 days) bursts of fluxes of low energy (45-300 keV) electrons and (300-750 $\mathrm{keV}$ ) protons with approximately 27-day periodicity at $1 \mathrm{AU}$, registered at SOHO and ACE during in the deep solar activity minimum in 2007-08, may be considered as particles associated with magnetic traps, carrying electrons, accelerated in Jupiter magnetosphere to the inner heliosphere. Regions of generation of low energy particles are magnetic field structures inside the 
traps with high level of turbulence appearing due to variations of solar wind speed. The electrons registered during the 2007-2008 solar activity minimum have two components: (a) periodic 27-day gradual Jovian-originated variations; (b) quasi-periodic short (2-3 days) increases of low-energy electrons and protons, accelerated directly inside the magnetic trap. Numerical simulations to model the propagation of energetic protons and electrons in the presence of such magnetic configuration are presented.

\section{Acknowledgements}

The work was supported by the Russian Foundation for Basic Research, № 14_02_00196.

\section{References}

[1] D.L. Chennet, T.F. Conlon, J.A. Simpson, J. Geophys. Res., 79 (1974) 3551.

[2] B.J. Teegarden et al, J. Geophys. Res., 79 (1974) 3615.

[3] S.M. Krimigis, E.T. Sarris, T.P. Armstrong, Geophys. Res. Lett., 2 (1975) 561.

[4] J. Giacalone, J.R. Jokipii, and J. Kota, Astrophys J., 573 (2002), 845.

[5] J. Kota and J.R. Jokipii, Geophys. Res. Lett., 18 (1991) 1797.

[6] J. Kota and J.R. Jokipii, Science, 268 (1995) 1024.

[7] R. Modzelewska and M.V. Alania, Solar Phys., 286 (2013) 593. 